\title{
Competitividad de operadores logísticos: un análisis bibliométrico en la base de datos Scopus, 2017 - 2021
}

\section{Competitiveness of logistics operators: a bibliometric analysis in the Scopus database, 2017 - 2021}

Elvira García-Huamantumba

Universidad Norbert Wiener, Lima, Perú

elvira1garcia2@gmail.com

https://orcid.org/0000-0001-7773-828X

Manuel Alberto Hidalgo-Tupia

Universidad Nacional Mayor de San Marcos, Lima, Perú

hidalgoman@gmail.com

iD https://orcid.org/0000-0002-9644-2406

María Mini Martin-Bogdanovich

Universidad Norbert Wiener, Lima, Perú

contactomaritamartinb@gmail.com

(D) https://orcid.org/0000-0002-1762-6339

Recepción: 02/08/2021 | Aceptación: 09/11/2021 | Publicación: 25/11/2021

Cómo citar (APA, séptima edición):

García-Huamantumba, E., Hidalgo-Tupia, M.A., y Martin-Bogdanovich, M.M. (2021). Competitividad de operadores logísticos: un análisis bibliométrico en la base de datos Scopus, 2017 - 2021. INNOVA Research Journal, 6(3.2), 92-117. https://doi.org/10.33890/innova.v6.n3.2.2021.1875

\section{Resumen}

El objetivo de la investigación fue realizar el análisis de la Competitividad de operadores logísticos a través de un estudio bibliométrico en la base de datos Scopus, 2017 - 2021. La estrategia de búsqueda consideró la selección de publicaciones en el idioma inglés de la base de datos Scopus. El algoritmo de búsqueda utilizado fue competitiveness of logistics operators, vinculado al título del artículo, resumen y palabras claves, se filtraron los siguientes tipos de documentos: article, book, book chapter, conference paper, editorial and review, fueron ordenados por su relevancia. El número de documentos seleccionados fue 148, con un total de 428 autores, de los cuales 404 registraron un documento y 24 autores registraron entre dos a más documentos, también se 
identificó 523 palabras claves. Se concluyó que las tendencias actuales de desarrollo a nivel mundial, se enfocan en el equilibrio entre el desarrollo sostenible económico, social y ambiental. La producción científica anual sobre el tema es aún escasa, habiéndose logrado en el año 2020 la cifra récord de 42 artículos, publicados en las revistas Sustainability (Switzerland), International Journal Of Logistics Management y Journal Of Cleaner Production. El documento de mayor citación es el de Ansari ZN (2017). Se identificó 404 autores que publicaron un artículo y sólo 2 autores han publicado 4 artículos cuyo aporte no llegan ni al 1\%. La mayor colaboración entre países es entre China, Reino Unido, Estados Unidos e India. Los temas de tendencias se orientan al Supply chain management.

Palabras claves: competitividad; operadores logísticos; innovación; bibliometría.

\begin{abstract}
The objective of the research was to perform the competitiveness analysis of logistics operators through a bibliometric study in the Scopus database, 2017 - 2021. The search strategy considered the selection of publications in the English language from the database Scopus. The search algorithm used was competitiveness of logistics operators, linked to the title of the article, abstract and keywords, the following types of documents were filtered: article, book, book chapter, conference paper, editorial and review, they were ordered by their relevance. The number of documents selected was 148, with a total of 428 authors, of which 404 registered one document and 24 authors registered between two or more documents, 523 keywords were also identified. It was concluded that current development trends worldwide focus on the balance between economic, social and environmental sustainable development. The annual scientific production on the subject is still scarce, having achieved a record number of 42 articles in 2020, published in the journals Sustainability (Switzerland), International Journal of Logistics Management and Journal of Cleaner Production. The document with the highest citation is Ansari ZN (2017). 404 authors were identified who published an article and only 2 authors have published 4 articles whose contribution does not even reach $1 \%$. The largest collaboration between countries is between China, the United Kingdom, the United States and India. The trend topics are oriented to Supply chain management.
\end{abstract}

Keywords: competitiveness; logistics operators; innovation; bibliometrics.

\title{
Introducción
}

La innovación es de vital importancia para la gestión de la cadena de abastecimiento internacional, el dilema innovador describe, como las empresas locales se benefician por la innovación, tal es así que les permite competir con las empresas multinacionales. Dentro de esta cadena, la globalización económica y el crecimiento del comercio mundial han permitido mayor interés por la competitividad de los operadores logísticos, debiendo considerar cada vez más los factores que influyen en los niveles de costos, calidad, velocidad, seguridad entre otros.

Desde el punto de vista académico, se resalta que la investigación sobre la competitividad de los operadores logísticos y la innovación, han acumulado conocimientos relevantes sobre la compleja dinámica de la competitividad logística en diversos entornos internacionales, sin embargo, existen diferentes puntos de vistas que explican dichas dinámicas. Al respecto, el 
problema de investigación trata sobre los vacíos y discrepancias que se presentan en la comprensión de la competitividad de los operadores logísticos.

En el afán de querer profundizar en la problemática de estudio, no se logró ubicar un estudio bibliométrico que informe sobre el avance temático y la evolución al respecto, por tal motivo es necesario que los especialistas en los negocios internacionales, conozcan los modelos que estuvieron vigentes como el enfoque logístico, información de sistemas, gestión de puertos, navieras, transporte de carga, toma de decisiones, entre otros, que no estaban integrados, por tal razón generaba atrasos, costos elevados e insatisfacción de los clientes mayoristas y más aún el mercado se quedaba desabastecido, motivando la producción de productos internos y sin mayor control de calidad, afectando a la competitividad de los operadores logísticos.

Sin embargo, el nuevo escenario económico ha obligado a la implementación del supply chain management, la robótica, inteligencia artificial, la big data, entre otras metodologías. Los modelos de desarrollo sostenible, se sustenta en un mayor interés por el cuidado del medio ambiente, la sostenibilidad, el desarrollo de los puertos verdes, la mayor orientación hacia el campo social, armonía en el desarrollo humano e industrial. A razón de la Covid-19 han de emerger nuevos modelos que son necesarios conocerlos y que la academia aporte para lograr a atender el mercado post pandemia sin descuidar los protocolos de seguridad de la vida humana para la preservación. Lo antes expuesto, permitirá a la vez diseñar curriculas con temáticas que atiendan a la realidad futura y por lo tanto profesionales que hagan competitivo la operación logística en el comercio internacional.

En consecuencia, el estudio se enfoca en lograr los siguientes objetivos específicos: (a) analizar la producción científica del 2017 al 2021 en el área de competitividad de los operadores logísticos; (b) conocer las fuentes más relevantes con producción científica 2017-2021; (c) Identificar los documentos más citados a nivel mundial; (d) Conocer la productividad del autor en concordancia con el número de documentos publicados; (e ) Conocer la colaboración entre países, instituciones y autores; (f) Identificar los temas nichos, motor, básico en declive y tendencia.

\section{Metodología}

La presente investigación desarrolla una revisión bibliométrica de la literatura científica sobre la competitividad de los operadores logísticos. La estrategia de búsqueda considera la selección de publicaciones en idioma inglés de la base de datos Scopus. La búsqueda realizada comprendió el período 2017-2021, del ámbito geográfico global. El algoritmo de búsqueda utilizado fue competitiveness of logistics operators, vinculado al título del artículo, resumen y palabras claves, filtramos los siguientes tipos de documentos: article, book, book chapter, conference paper, editorial and review. Las publicaciones se ordenaron por su relevancia considerando el número de citas obtenidas.

La revisión se realizó a través de la bibliometría que fue descriptiva y retrospectiva, empleando el software Bibliometrix y el paquete estadístico R-Studio. Con fecha 07-07-2021 se realizó la última búsqueda, encontrándose 148 documentos, con un total de 428 autores, de los cuales 404 registraron un documento y 24 autores registraron entre dos a más documentos; también se identificó 523 palabras claves, las cuales se descargaron en formato Bid Text. Esta fuente de 
consulta reconocida se utilizó para identificar el número total de publicaciones indexadas en la base de datos Scopus (ver tabla 1).

Destaca la publicación de artículos y artículos de conferencia, entre los años 2017 y 2021 la mayor producción científica fue en el año 2020 con 42 publicaciones, la fuente de mayor relevancia correspondió a Sustainability (Switzerland) con 13 publicaciones.

\section{Tabla 1}

Información general de la búsqueda en Scopus

\begin{tabular}{|c|c|}
\hline Descripción & Resultados \\
\hline \multicolumn{2}{|l|}{ MAIN INFORMATION ABOUT DATA } \\
\hline Timespan & 2017:2021 \\
\hline Sources (Journals, Books, etc) & 90 \\
\hline Documents & 148 \\
\hline Average years from publication & 1.95 \\
\hline Average citations per documents & 11.41 \\
\hline Average citations per year per doc & 3.169 \\
\hline References & 1 \\
\hline \multicolumn{2}{|l|}{ DOCUMENT TYPES } \\
\hline Article & 109 \\
\hline Book & 6 \\
\hline book chapter & 4 \\
\hline conference paper & 6 \\
\hline Editorial & 1 \\
\hline Review & 22 \\
\hline \multicolumn{2}{|l|}{ DOCUMENT CONTENTS } \\
\hline Keywords Plus (ID) & 523 \\
\hline Author's Keywords (DE) & 600 \\
\hline \multicolumn{2}{|l|}{ AUTHORS } \\
\hline Authors & 428 \\
\hline Author Appearances & 461 \\
\hline Authors of single-authored documents & 14 \\
\hline Authors of multi-authored documents & 414 \\
\hline \multicolumn{2}{|l|}{ AUTHORS COLLABORATION } \\
\hline Single-authored documents & 14 \\
\hline Documents per Author & 0.346 \\
\hline Authors per Document & 2.89 \\
\hline Co-Authors per Documents & 3.11 \\
\hline Collaboration Index & 3.09 \\
\hline
\end{tabular}

Fuente: Información en base a Scopus (07 de julio 2021) 


\section{Revisión de la literatura}

De la revisión de la literatura efectuada, se consideró importante involucrar las definiciones de los términos más relevantes del presente artículo, competitividad, operador logístico, innovación, sostenibilidad.

Según Botero (2014), entiende por competitividad a la "capacidad de una organización pública o privada, lucrativa o no, de mantener sistemáticamente ventajas que le permitan alcanzar, sostener y mejorar una determinada posición en el entorno socioeconómico" (p. 188).

Según Vega, Flores, y Solis (2011), como se citó en Botero (2014):

La ventaja de una organización estaría en la habilidad, recursos, conocimientos y atributos de los que dispone, los mismos de los que sus competidores carecen o tienen en menor medida, lo cual hace posible la obtención de rendimientos superiores a los de ellos (p. 188).

Asimismo, Corona (2002), como se citó en Botero (2014), "La competitividad es una variable multifactorial que se compone de formación empresarial, prácticas administrativas, laborales y productivas, la innovación (interna o externa) y el progreso tecnológico” (p. 188).

Según Consejo Nacional de Competitividad (2018), como se citó en (Calatayud y Montes, 2021), América Latina y el Caribe (ALC) presentó un importante rezago en el desempeño logístico, frente a otras regiones. "Indicadores internacionales dan cuenta que ALC califica sistemáticamente por debajo de los niveles de las economías avanzadas, a la vez que su propia calificación ha retrocedido en los últimos lustros" (p. 15).

Según la ANADIF (2001), como se citó en Granillo-Macías (2019) precisó que un operador logístico, es aquella empresa que por encargo de su cliente diseña los procesos de una o varias fases de su cadena de suministro, organiza, gestiona y controla dichas operaciones utilizando para ello infraestructuras físicas, tecnología y sistemas de información, propios o ajenos, independientemente de que preste o no los servicios con medios propios o subcontratados.

Según Llinás (1997) como se citó en Botero (2014) "La concepción moderna consiste en que el empresario debe crear los factores para que estos permitan altos niveles de eficiencia o competitividad. Estos factores modernos son: la tecnología, la información, la infraestructura, el conocimiento y la estrategia." (p. 191).

El continuo crecimiento del comercio mundial depende de la eficiencia de las estructuras de soporte logístico tales como los servicios logísticos. Los efectos se ven mayormente en las exportaciones. La principal implicación en política económica es que la inversión continua en infraestructura y servicios logísticos pueden impactar positivamente en el comercio internacional (Gani, 2017).

Según Knop (2019) los sectores de trasportes y operadores logísticos, que aplicaron el modelo SERQUAL ponderado y no ponderado "para medir la calidad del servicio, mostraron 
como resultado las debilidades y oportunidades de mejora respectivas. En dicho caso, se requiere relacionar explícitamente la calidad de servicio con la competitividad" (p. 151).

El florecimiento del comercio electrónico contribuyó a la enorme demanda para desarrollar instalaciones logísticas dedicadas a procesar órdenes de compra on - line. Sin embargo, no se ha estudiado suficientemente como el uso de la tierra por la logística cambió para acomodar la nueva atención de pedidos, que estudian el caso de Shezhen, el "hub" chino de atención de pedidos. La atención de los pedidos en línea actuó como catalizador para determinar cómo las instalaciones logísticas son diseñadas, re - desarrolladas, relocalizadas y utilizadas para convenir con los cambiantes requerimientos en la administración de la cadena de suministros (Xiao, Yuan, Sun, y Sun, 2021).

Para desarrollar los procesos de innovación en la gestión y lograr la sostenibilidad ambiental dentro de la industria portuaria. Di Vaio, Varriale, y Alvino (2018) sugieren considerar y utilizar los instrumentos de gestión, como el Balanced Scorecard (BSC) y el Tableau de Bord $(\mathrm{TdB})$, y recomiendan los sistemas de capacitación para todos los actores del puerto, incluidos los cargadores, transportistas o instituciones responsables, como las autoridades portuarias, tomen decisiones con respecto a la gestión eficaz y eficiente de los recursos ambientales.

Sin embargo, también destaca las limitaciones del marco legislativo, que no es capaz de proporcionar e implementar con éxito estos instrumentos para la sostenibilidad ambiental de los puertos y propone soluciones de gestión específicas de acuerdo con las perspectivas de innovación en la gestión con el objetivo de desarrollar puertos verdes competitivos (Di Vaio, 2018, p. 26).

Según Bailey y Solomon (2004), como se citó en Di Vaio (2018) sostienen que la contaminación ambiental de los barcos se debe a menudo a sus características técnicas. Por ejemplo, la contaminación del aire es generada por motores diésel que queman combustible con alto contenido de azufre, conocido como aceite de combustible; otros contaminantes incluyen dióxido de azufre (SO 2), óxido de nitrógeno (NO x) (p. 4).

Para ser competitivo se requiere un enfoque conjunto entre el puerto y el resto de autoridades y empresas, creando las condiciones para el desarrollo sostenible.

La Administración de la cadena sostenible de suministros - ACSS - atrae un considerable interés tanto de académicos como de profesionales, a juzgar por el crecimiento exponencial de las publicaciones científicas al respecto (Dubey, Gunasekaran, Childe, Papadopoulos \& FossoWamba, 2017). Ha habido una transición desde el concepto de Cadena sostenible de suministro (CSS), la administración de la cadena sostenible de suministros (ACSS), hasta la propuesta de Administración de la cadena sostenible de suministros de clase mundial (ACSSCM). La propuesta de los autores es una ACSSCM basada en una red o entramado.

Sobre la definición de sostenibilidad hallada en la literatura sobre cadena de suministro, Dubey, Gunasekaran, Childe, Papadopoulos, y Fosso-Wamba (2017) plantean al inicio la necesidad de una desambiguación entre ACS Verde, ACS Ambiental, ACS Sostenible, así como CS Ambiental, CS ética, y CS Responsable. Además de aportar un esquema de clasificación de la 
literatura sobre ACSS. Aporta asimismo la identificación y clasificación de los facilitadores o habilitadores (enablers) de la ACSS, aportando a continuación las "brechas de investigación". Finalmente, aporta un marco conceptual del ACSSCM con 6 pilares: Ambiental, Valores sociales y ética; estabilidad económica, evaluación de la performance operacional, factores internos y factores externos.

Por su lado, Calatayud y Montes (2021) sostuvieron, "Siendo el transporte uno de los principales contribuyentes a las emisiones de gases de efecto invernadero, la logística también posee un rol en la desaceleración del cambio climático" (p. 12). La diversificación de los flujos comerciales hacia sectores más sofisticados ha sido uno de los objetivos de las políticas de integración de la región de las últimas décadas. Sin embargo, el Informe Especial sobre Integración y Comercio del BID, "La integración económica no fue tan eficaz en promover la competitividad". (Giordano, Campos, Ortiz, Michalczewsky, y DeAngelis, 2018, p. 80).

La bibliometría facilita la revisión sistemática de la literatura con énfasis en el análisis de las teorías, contextos, características, metodologías, y oportunidades para futuras investigaciones en este campo, como es el caso de las revistas científicas.

\section{Resultados}

A nivel mundial, las revistas científicas y los medios de comunicación en el área de la competitividad de los operadores logísticos han experimentado un incremento significativo durante los últimos años (ver figura 1).

\section{Figura 1}

Producción científica anual de Artículos (2017-2021)

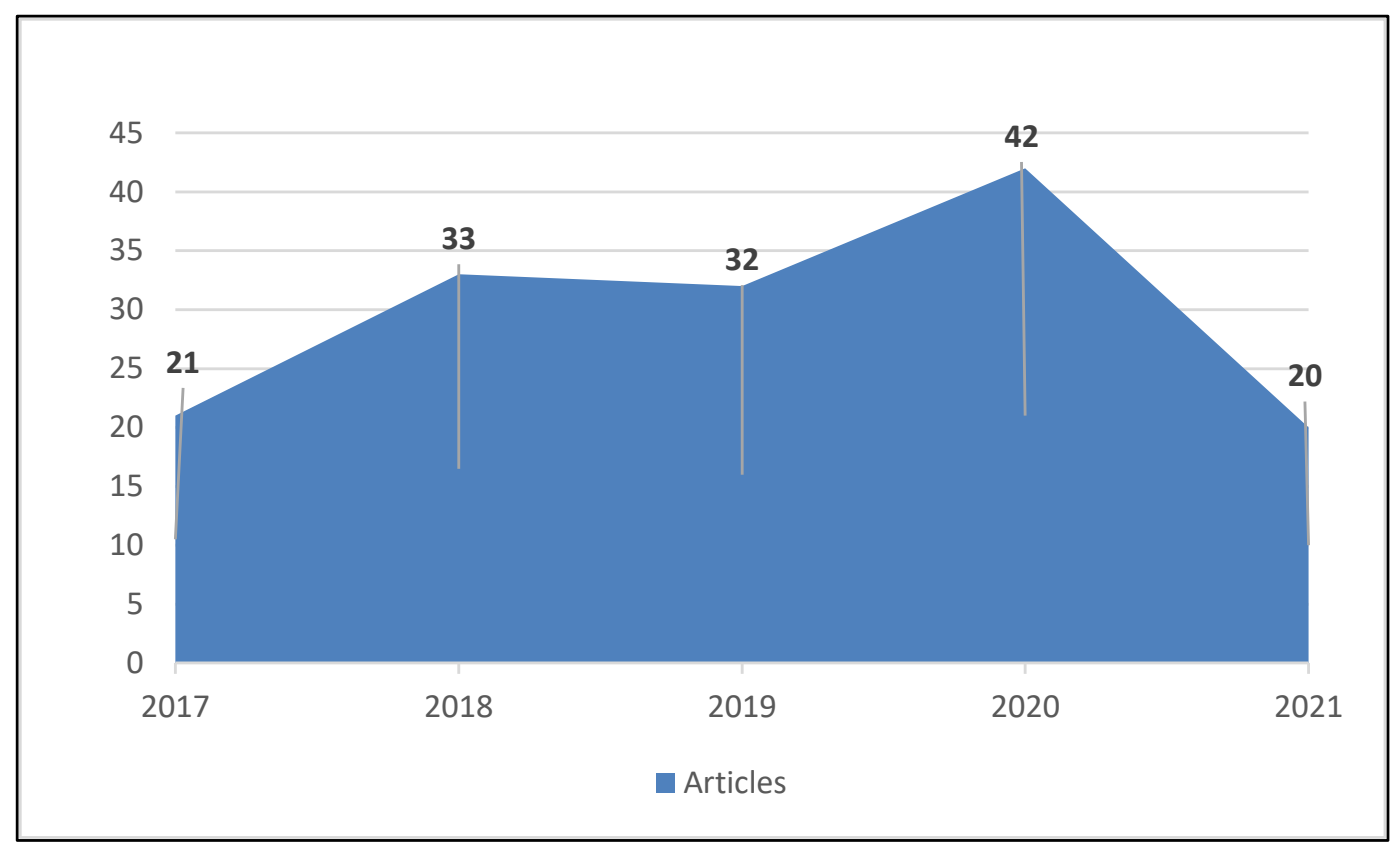

Fuente: Información en base a Scopus (07 de julio 2021) 
La Fuentes más relevantes de producción científica, fueron lideradas por la revista Sustainability (Switzerland) con 13 publicaciones, que significa el 17\% de las 20 revistas con mayor publicación, seguido por International Journal of Logistics Management con 7 publicaciones, Journal of Cleaner Production con 6 publicaciones, Benchmarking, International Journal of Logistics Systems And Management, International Journal Of Shipping And Transport Logistics, International Journal Of Supply Chain Management y Transport Reviews cada una de ellas con 4 publicaciones, las demás con tres a menos publicaciones. La suma total de estas 8 revistas, significa el $61 \%$ del total, (ver tabla 2 ).

\section{Tabla 2}

Fuentes más relevantes con producción científica (2017-2021)

\begin{tabular}{lcc}
\hline Fuentes & Artículos & $\%$ \\
\hline Sustainability (Switzerland) & 13 & $17 \%$ \\
International Journal Of Logistics Management & 7 & $9 \%$ \\
Journal Of Cleaner Production & 6 & $8 \%$ \\
Benchmarking & 4 & $5 \%$ \\
International Journal Of Logistics Systems And Management & 4 & $5 \%$ \\
International Journal Of Shipping And Transport Logistics & 4 & $5 \%$ \\
International Journal Of Supply Chain Management & 4 & $5 \%$ \\
Transport Reviews & 4 & $5 \%$ \\
International Journal Of Logistics Research And Applications & 3 & $4 \%$ \\
Maritime Economics And Logistics & 3 & $4 \%$ \\
Maritime Policy And Management & 3 & $4 \%$ \\
Transportation Research Part A: Policy And Practice & 3 & $4 \%$ \\
Transportation Research Part E: Logistics And Transportation & & \\
Review & 3 & $4 \%$ \\
Case Studies On Transport Policy & 2 & $3 \%$ \\
International Journal Of Physical Distribution And Logistics & & \\
Management & 2 & $3 \%$ \\
International Journal Of Production Research & 2 & $3 \%$ \\
Journal Of Manufacturing Technology Management & 2 & $3 \%$ \\
Operations Research/ Computer Science Interfaces Series & 2 & $3 \%$ \\
Research In Transportation Business And Management & 2 & $3 \%$ \\
Review Of International Business And Strategy & 2 & $3 \%$ \\
Total & 75 & \\
\hline
\end{tabular}

Fuente: Información en base a Scopus (07 de julio 2021)

Las principales publicaciones más citadas fueron de Ansari Zn, 2017, J Clean Prod con un total de 144 citas, seguido por De Oliveira Ur, 2018, J Clean Prod con 108 citaciones, y en tercer lugar Dubey R, 2017, Int J Logist Manage con 80 citaciones; los tres significan el $31 \%$ del total de las 20 principales publicaciones más citadas. (ver figura 2). 


\section{Figura 2}

Documentos más citados a nivel mundial (2017-2021)

\begin{tabular}{|c|c|}
\hline ANSARI ZN, 2017, J CLEAN... & 144 \\
\hline DE OLIVEIRA UR, 2018, J CLE... & 108 \\
\hline DUBEY R, 2017, INT J LOGIST... & 80 \\
\hline CRAINIC TG, 2018, EUR J OPE... & 73 \\
\hline KAZANCOGLU Y, 2018, J CLEA... & 72 \\
\hline YILDIZ ANKAYA S, 2019, J... & 67 \\
\hline TARASOVA VV, 2017, CHAOS... & 58 \\
\hline EVANGELISTA P, 2018,... & 52 \\
\hline DI VAIO A, 2018,... & 52 \\
\hline PAROLA F, 2017, TRANSP REV & 48 \\
\hline DI VAIO A, 2018, ENERGY... & 47 \\
\hline KLUMPP M, 2018, INT J LOGIS... & 43 \\
\hline LO SM, 2018, J CLEAN PROD & 34 \\
\hline CHEN Y, 2020, J IND INTEGR... & 32 \\
\hline AGAMEZ-ARIAS ADM, 2017, ... & 30 \\
\hline JIAN J, 2019, SUSTAINABILITY & 27 \\
\hline YANG Y, 2019, SUPPLY CHAIN... & 27 \\
\hline YANG CS, 2017, INT J PHYS... & 26 \\
\hline ALSAAD AK, 2018, EUROMED... & 25 \\
\hline SAHU AK, $2017, \ldots$ & 23 \\
\hline
\end{tabular}

Fuente: Información en base a Scopus (07 de julio 2021)

Según la Ley Lotka, el $94.4 \%$ de autores han publicado un documento, el $4 \%$ de autores, han publicado 2 documentos, el $1.2 \%$ de autores han publicado 3 documentos y el $0.5 \%$ de autores, han publicado 4 documentos. (ver tabla 3)

\section{Tabla 3}

Productividad del autor a través de la ley Lotka

\begin{tabular}{ccc}
\hline Documentos publicados & $\mathbf{N}^{\circ}$ de Autores & \% por autor \\
\hline 1 & 404 & 0,944 \\
2 & 17 & 0,04 \\
3 & 5 & 0,012 \\
4 & 2 & 0,005 \\
\hline
\end{tabular}

Fuente: información en base a Scopus (07 de julio 2021)

El autor principal con 4 publicaciones científicas es Parola F. 2017, con el documento "The Drivers Of Port Competitiveness: A Critical Review", representa el 3\% del total de documentos publicado del Scopus, en el campo de la competitividad de los operadores logísticos. 
El trabajo colaborativo para los artículos científicos, entre países se da con mayor frecuencia entre: China. EEUU, Reino Unido, Hong Kong y Bélgica y entre: EEUU, India, Corea y Francia; seguidos por Australia y Singapur, Suecia e Italia, Alemania y Países Bajos. (ver figura 3).

\section{Figura 3}

Mapa de colaboración entre países

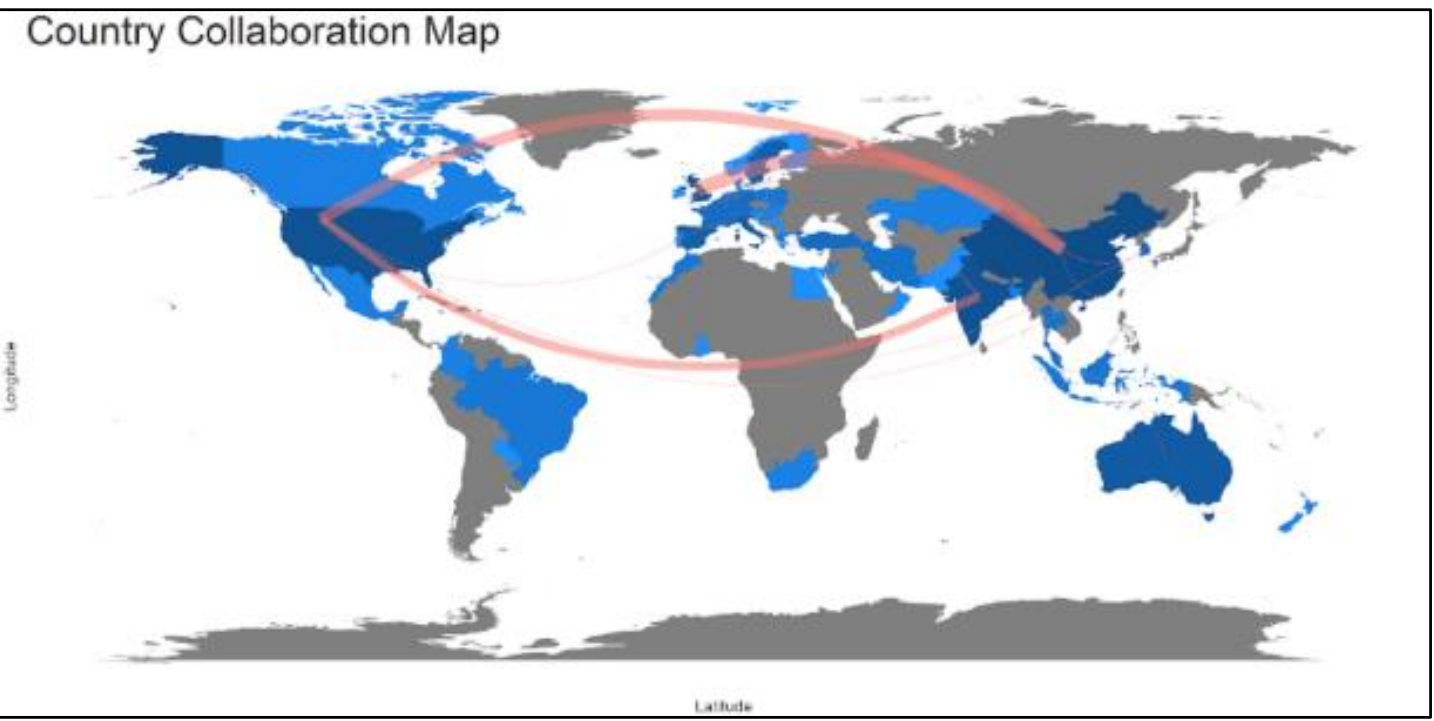

Fuente: Información en base a Scopus (07 de julio 2021)

Sin embargo, el tema colaborativo también es notorio entre instituciones y autores (ver figura 4).

\section{Figura 4}

Mapa de colaboración entre instituciones y autores

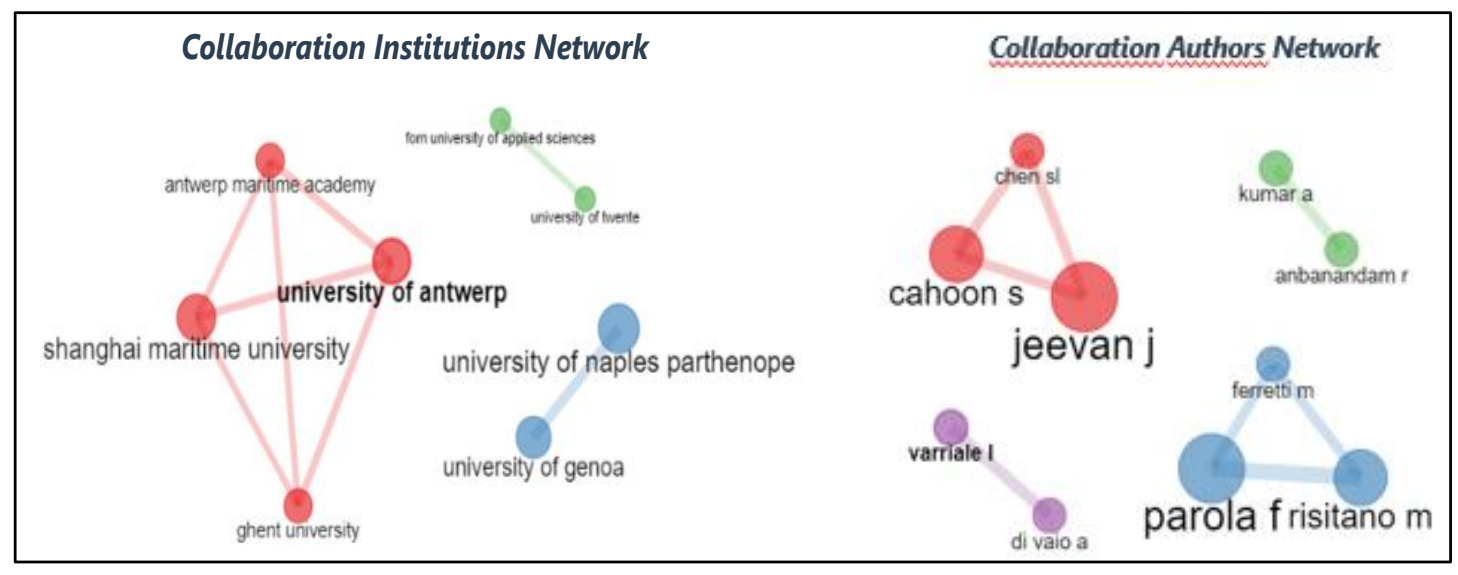

Fuente: información en base a Scopus (07 de julio 2021) 
En la figura 5 se aprecia el mapa temático de palabras claves, agrupadas e identificadas en un plano cartesiano, donde la relevancia del grado de centralidad está ubicada en el eje de abscisa y se cruza con la densidad de grado de desarrollo ubicada en el eje de ordenadas, formando 4 cuadrantes: en el cuadrante I, se posesionan los términos motores o impulsores relacionados a competitividad de operadores logísticos, donde destaca (logística, revisión de literatura, competitividad, marco conceptual, transporte de carga, innovación, transporte marítimo, puertos, trabajo de investigación y análisis de demanda. En el cuadrante II se posesionan los términos nicho, relacionados a competitividad de operadores logísticos, donde destaca (toma de decisiones, desarrollo sostenible, cadena de suministros, puertos, buques, competencia, proceso de toma de decisiones, transporte de carga, redes complejas, sistemas de información). En el cuadrante IV se posesionan los términos básicos, relacionados a competitividad de operadores logísticos, donde destaca (gestión de la cadena de suministro, sostenibilidad, operación portuaria, comercio, envío, partes interesadas, gestión de la cadena de suministro verde, revisiones de la literatura, marketing, rentabilidad), como podemos observar las proyecciones del segmento entre el origen y los puntos del tercer cuadrante, en su proceso de traslación, no detecta temas emergentes o en declive.

\section{Figura 5}

\section{Mapa temático de palabras clave, con base Scopus}

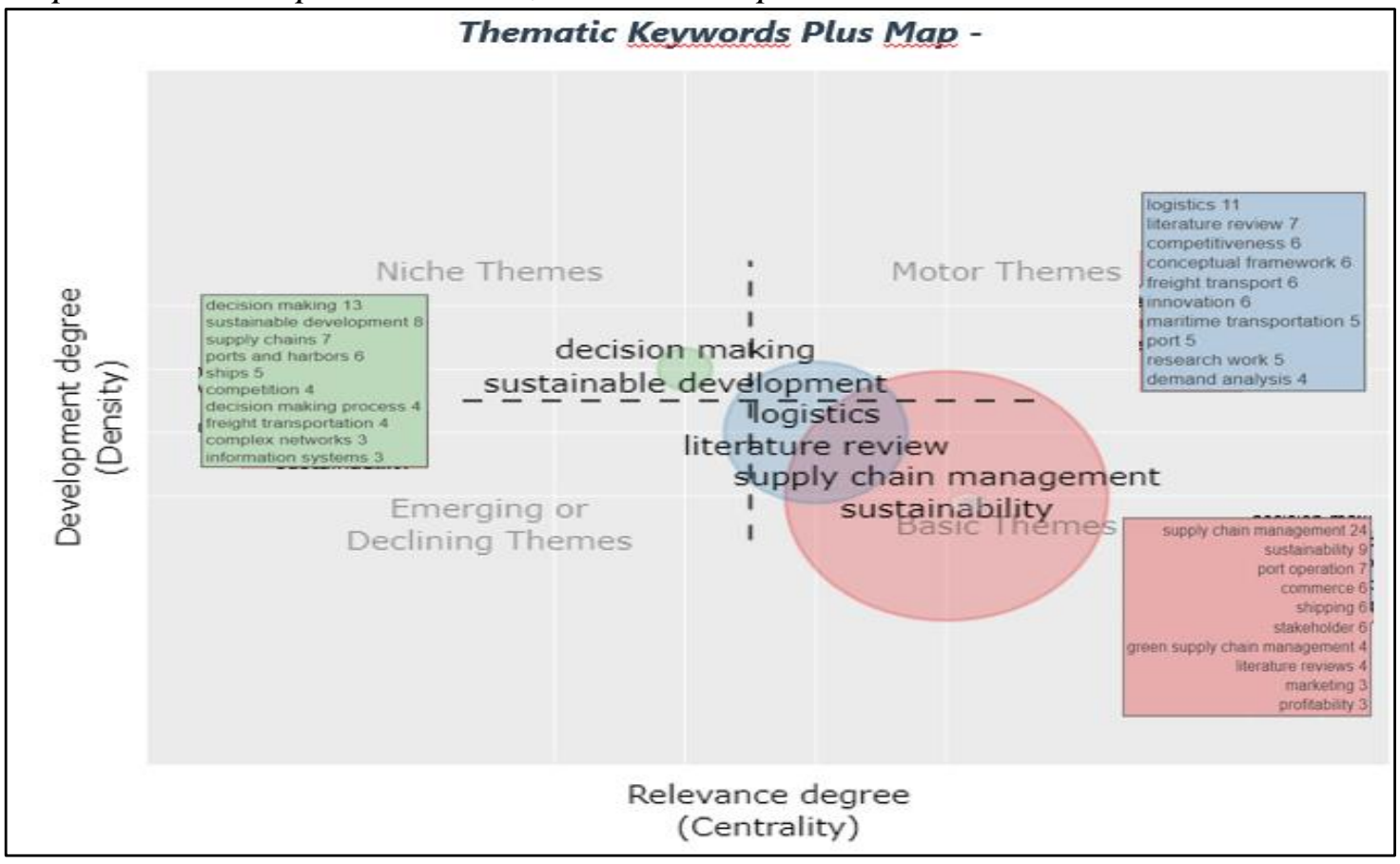

Fuente: Información en base a Scopus (07 de julio 2021)

Los temas de tendencia más relevantes son gestión de la cadena de suministro, toma de decisiones, logística, sostenibilidad, desarrollo sostenible, revisión de la literatura, operación portuaria, cadenas de suministro, comercio y competitividad, en ese orden (ver tabla 4). Información de orientación para futuras investigaciones. 


\section{Tabla 4}

Temas de tendencia (2017-2021)

\begin{tabular}{lcc}
\hline Item & Freq & Year \\
\hline Supply Chain Management & 24 & 2019 \\
Decision Making & 15 & 2019 \\
Logistics & 11 & 2019 \\
Sustainability & 9 & 2018 \\
Sustainable Development & 9 & 2018 \\
Literature Review & 7 & 2018 \\
Port Operation & 7 & 2019 \\
Supply Chains & 7 & 2018 \\
Commerce & 6 & 2020 \\
Competitiveness & 6 & 2018 \\
Conceptual Framework & 6 & 2018 \\
Freight Transport & 6 & 2020 \\
Innovation & 6 & 2018 \\
Ports And Harbors & 6 & 2019 \\
Shipping & 6 & 2020 \\
Stakeholder & 6 & 2018 \\
Research Work & 5 & 2019 \\
Ships & 5 & 2020 \\
Competition & 4 & 2020 \\
Demand Analysis & 4 & 2020 \\
Freight Transportation & 4 & 2020 \\
Profitability & 4 & 2020 \\
\hline
\end{tabular}

Fuente: información en base a Scopus (07 de julio 2021)

Como se puede apreciar en la figura 6, el uso con mayor énfasis se da en "supply chain management" en comparación con el siguiente término relacionado, "logistic". Es notable asimismo que los términos ligados a "transporte" ("shipping", "maritime transportation", "freight transportation", "transportation economics", "transportation planning"; "international transportation", "transportations system") se han diversificado notablemente. Asimismo, es notable el énfasis en la toma de decisiones ("decision making") y lo relacionado a la sostenibilidad ("sustainability" y "sustainable development")

El cambio es más que semántico; implica un cambio conceptual, que redefine la acción humana en la actividad especifica. Al cambiar de usar "logística" a "administración de la cadena logística”, se está reflejando tanto un cambio en el contexto, más como reflejo de la globalización, como resultado de la propia respuesta organizacional y su mayor interés como materia de investigación. 


\section{Figura 6}

Variación en el Énfasis de constructo según el Bibliometrix con base Scopus

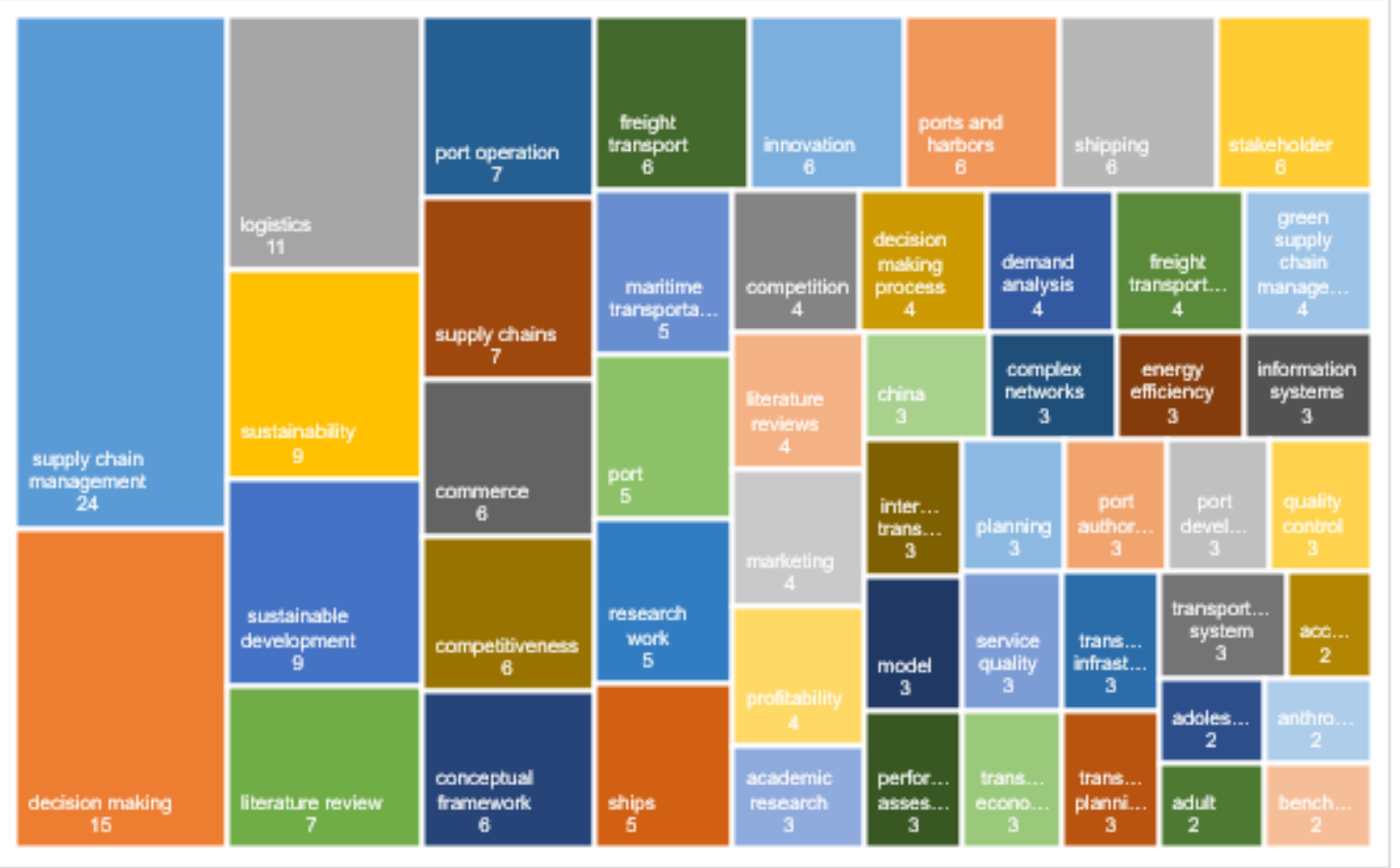

Fuente: Información en base a Scopus (07 de julio 2021)

Por tanto, los resultados muestran el comportamiento histórico de los medios categorizados por continentes; los países con mayor cantidad de literatura; el número total de revistas por cuartil; la producción de los autores a lo largo del tiempo; la producción anual; la producción científica por país, los países más citados; las ciudades más relevantes por autor; las palabras más frecuentes; los documentos más citados a nivel mundial; las afiliaciones más relevantes; las fuentes más relevantes; las fuentes más dinámicas; las fuentes de impacto; los temas de tendencia; el impacto del autor; y además identifican algunos indicadores que pueden influir en la producción de investigación en el campo de estudio científicos indexadas en Scopus.

\section{Discusión}

En el presente estudio se hace una revisión exhaustiva de 148 publicaciones académicas sobre la competitividad de los operadores logísticos, que fueron publicados entre el 2017 al 2021, estas publicaciones fueron clasificados desde distintas perspectivas. La discusión del presente estudio lo enfocaremos desde la perspectiva de los temas más frecuentes abordados en las publicaciones revisadas y de las revistas más relevantes. Entre los temas más frecuentes se presentan: 


\section{Gestión de la Cadena de Suministros - SCM}

La gestión de la cadena de suministros (supply chain management - SCM), que es un tema que se presenta en 24 publicaciones, fue el que apareció con más frecuencia en las publicaciones de nuestra revisión bibliométrica. Según Ansari y Kant (2017), De-Oliveira, Espindola, da Silva, da Silva y Rocha (2018), Dubey, Gunasekaran, Childe, Papadopoulos y Wamba (2017), Klumpp (2018), Lo, Zhang, Wang, y Zhao (2018), Dubey, Gunasekaran, Papadopoulos, Childe, Shibin, y Wamba (2017), Abushaikha, Al-Weshah, y M. (2020), entre otros, consideran que la gestión de la cadena de suministros - SCM, se define como la gestión de los materiales, la gestión de la información y la gestión de los flujos de capitales, en todas las etapas de la cadena desde la fuente primaria de los recursos hasta el consumidor final.

Según Pandey, Singh, Gunasekaran y Kaushik (2020) una cadena de suministros es una red de diferentes organizaciones, alineadas a través de vínculos ascendentes y descendentes con organizaciones de terceros, proveedores, consumidores y socios, desde el punto de origen hasta el punto de consumo. Las cuales, mediante el flujo de bienes, servicios e información relacionada, producen un valor superior para el cliente a un menor costo para la cadena de suministros en su conjunto, entregando el producto correcto, en la cantidad correcta, en el lugar correcto en el momento correcto, para el cliente correcto al costo correcto.

Según Russell, Lee, y Clift (2018) los objetivos de desarrollo sostenible deben cumplir con tres conjuntos de restricciones: eficiencia tecno-económica, compatibilidad ambiental y equidad social. Asimismo, la responsabilidad en la gestión de la cadena de suministro implica no solo operar dentro de los límites ecológicos, sino también debe incluir la equidad social transmitiendo beneficios en ambas direcciones. Por lo tanto, el consumo y la producción sostenibles solo pueden lograrse plenamente si se comparten los objetivos de desempeño (económicos, ambientales y sociales) y los principios de comportamiento (calidad), lo que lleva a objetivos, metas y requisitos comunes en toda la red de la cadena suministro que genera valor.

\section{Toma de Decisiones}

En segundo lugar, con una frecuencia de 15 publicaciones se presenta el tema toma de decisiones (decisión making). Según Kondratowicz, (1990); Vaio (2018); Di Vaio, Varriale, y Alvino (2018), definen la toma de decisiones como la elección de una opción de solución entre las distintas alternativas posibles, destacando la importancia de los instrumentos de gestión, en especial los instrumentos informáticos, que son un importante apoyo a la toma de decisiones.

"Diseñar un sistema de distribución física en mercados emergente, requiere ajustes continuos, porque las condiciones del mercado, competitivas y de transporte cambian constantemente; son restricciones comunes a las que tienen que enfrentarse las empresas multinacionales en muchos mercados emergentes", Boojihawon, Richeri, Liu, y Chicksand (2020). Como tal, la capacidad de las empresas multinacionales para capturar oportunidades depende de sus capacidades de agilidad estratégica, es decir, de la rapidez con la que puedan adaptar sus estrategias de RTM, aprender sobre la marcha desde cero y ser flexibles en responder a las necesidades de los clientes nuevos y existentes en esos mercados. 


\section{Logística}

La logística (logistics) estuvo en el tercer lugar con una frecuencia de repetición en 11 publicaciones. Según Klumpp (2018), existen tres tendencias académicas de investigación que han dominado la logística y la gestión de la cadena de suministros en las últimas tres décadas: (1) la integración y optimización general de las cadenas de suministro cada vez más globales, (2) cadenas de suministro ágiles, flexibles y resilientes, con el fin de mitigar el riesgo y la volatilidad de los impactos del mercado global y los clientes cada vez más exigentes, (3) operaciones de cadena de suministro y logística sostenibles.

En la cadena de suministro global, según Chen, Cheung, Chu y Xu (2017), la logística de carga global implica múltiples nodos logísticos, la conectividad de estos nodos se vuelve cada vez más crucial para planificar eficazmente estos envíos. Asimismo, la selección de un centro de transbordo, cada vez adquiere mayor importancia para el control de costos y la confiabilidad del servicio, y tiene que preocuparse por la conectividad con otros puertos que por la eficacia de un solo puerto.

\section{Sostenibilidad y Desarrollo Sostenible}

En el cuarto y quinto lugar se encontraron la sostenibilidad (sustainability) y el desarrollo sostenible (sustainable development), cada una con una frecuencia de 9 publicaciones estos dos temas que son similares tienen en total 18 publicaciones, solo superadas por la gestión de la cadena de suministros. Según Ansari y Kant (2017); Dubey, Gunasekaran, Childe, Papadopoulos y Wamba (2017); Vaio (2018); Zheng, Zhao, y Shao (2020); Mohammadi, Talaie, y Safari (2018), Según Jian, Guo, Jiang, An y Su (2019), consideran que la gestión sostenible de la cadena de suministros (SSCM) se encuentra en la intersección de los tres pilares básicos de la sostenibilidad como son el desempeño ambiental, desempeño social y el desempeño económico. Ansari y Kant (2017), considera que el campo de investigación sobre la gestión de la cadena de suministros sostenible (SSCM) está dominado por estudios cualitativos. Asimismo, Vaio (2018), consideran que la mayoría de los estudios sobre temas ambientales tienen que ver con procesos de fabricación, existen muy pocos estudios en servicios; además para (Jian, Guo, Jiang, An y Su (2019), la mayoría de los estudios analizados se enfocan principalmente en los objetivos económicos, muy pocos se enfocan en objetivos ambientales, en cambio en un nivel práctico para tomar decisiones las empresas recurren frecuentemente a la economía y el ambiente. 


\section{Figura 7}

\section{Los pilares de la sostenibilidad}

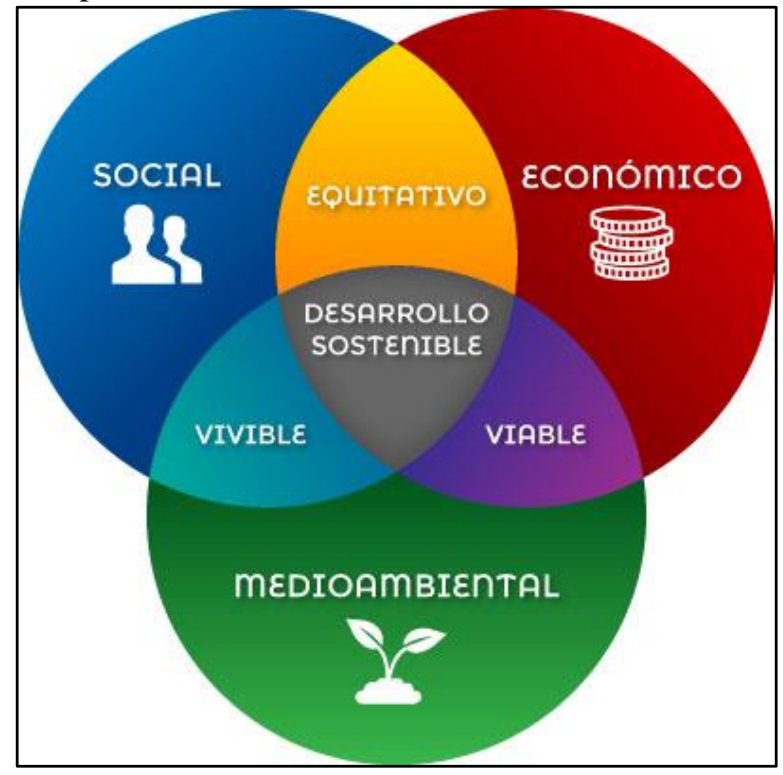

Fuente: https://www.regionalpymezonasur.org.ar/desarrollo-sostenible/ (Regional, 2019)

Según Srivastava (2007), la gestión de la cadena de suministros verde (GrSCM) se define como:

La integración del pensamiento ambiental en la gestión de la cadena de suministro, incluido el diseño del producto, el suministro y la selección de materiales, los procesos de fabricación, la entrega del producto final a los consumidores y la gestión final del producto después de su vida útil (pp. 54-55). La afirmación anterior fue sostenida también por De-Oliveira, Espindola, da Silva, da Silva y Rocha (2018)

Según Dubey, Gunasekaran, Childe, Papadopoulos y Wamba (2017), propone que la gestión de la cadena de suministros sostenible de clase mundial (WCSSCM) es un constructo que incluye seis dimensiones, dimensión ambiental, dimensión ética y de valores sociales, dimensión de estabilidad económica, dimensión de evaluación de desempeño operativo, dimensión de factores internos, dimensión de factores externos.

Sin embargo, Sibel y Bulent (2019) habla de 8 dimensiones, de la gestión de la cadena de suministro verde (GSCM) en el desempeño económico, ambiental y social, que son las tres dimensiones de la sustentabilidad corporativa. "Las ocho dimensiones que consideran son: compras, ecológicas, fabricación ecológica, distribución ecológica, envases ecológicos, marketing ecológico, educación ambiental, gestión ambiental interna y recuperación de inversiones” (p. 98).

\section{Revisión de literatura}


En el sexto lugar se encontraron la revisión de literatura (literature review) como tema recurrente con una frecuencia de 7 publicaciones. Estas publicaciones aplicaron amplias revisiones de literatura para abordar interesantes temas de la competitividad de los operadores logísticos, entre ellos estuvo Ansari y Kant (2017); De-Oliveira, Espindola, da Silva, y Rocha (2018); Dubey, Gunasekaran, Childe, Papadopoulos y Wamba (2017); Evangelista, Santoro y Thomas (2018); Parola, Risitano, Ferretti y Panetti (2017); Agamez-Arias, y Moyano-Fuentes (2017); Srivastava (2007).

\section{Las operaciones portuarias}

Las operaciones portuarias estuvieron en el séptimo lugar con una frecuencia de repetición en 7 publicaciones. Según Parola, Pallis, Risitano y Ferretti (2018), los puertos son una de las partes fundamentales en la cadena de suministros y las autoridades portuarias mantienen hoy una posición dominante en la gobernanza y organización portuaria. Estos entornos de gobernanza reformados estimulan a las autoridades portuarias a emprender estrategias de crecimiento y explorar nuevas oportunidades comerciales esforzándose por lograr objetivos más amplios y fortalecer funciones que en el pasado parecían poco convencionales para una institución pública.

Según Parola, Pallis, Risitano y Ferretti (2018), para las autoridades portuarias las actividades de responsabilidad social corporativa son más evidentes que nunca, al igual que el lanzamiento de proyectos e inversiones en países de ultramar, que se ven como una fuente adicional de ingresos y una oportunidad para explotar los recursos y capacidades desarrollados en casa, comprometiéndose activamente en proyectos logísticos fuera de sus límites físicos, para reforzar las cadenas de suministro y su propia competitividad.

Según Vaio (2018), los responsables de formular políticas de gestión portuaria, deben priorizar el tema ambiental adoptando una orientación proactiva hacia la reducción del impacto ambiental en el puerto

\section{Las cadenas de suministro}

Las cadenas de suministro estuvieron en el octavo lugar con una frecuencia de repetición en 7 publicaciones. Según Venkatesh y Bala (2012); Chaeet al. (2005), como se citó en Alsaad, Yousif y AlJedaiah (2018), sugieren que las ventajas competitivas en la cadena de suministro no solo dependen de la sinergia mutua de recursos es decir de origen interno, sino también de la colaboración conjunta entre socios comerciales, es decir de origen externo.

Según Klumpp (2018); Belhadi, Venkatesh, Kamble, Rehman, Verma, (2021) existen tres tendencias académicas de investigación que han dominado la logística y la gestión de la cadena de suministros en las últimas tres décadas: (1) La integración y optimización general de las cadenas de suministro cada vez más globales, (2) cadenas de suministro ágiles, flexibles y resilientes, con el fin de mitigar el riesgo y la volatilidad de los impactos del mercado global y los clientes cada vez más exigentes, (3) Operaciones de cadena de suministro y logística sostenibles.

Según Notteboom, Parola, Satta, y Risitano (2017), un centro logístico es un área territorial que tiene un equipamiento logístico y desarrolla actividades logísticas, siendo estos una parte importante en la cadena de suministros. 
Según Yang, Jia, y Xu (2019); las investigaciones sobre el aprendizaje de la cadena de suministro son de cuatro tipos: (1) orientada a procesos, (2) orientada a estructuras, (3) orientada a consecuencias y (4) otras definiciones informales. Sugiriendo que el aprendizaje en la cadena de suministros consta de capacidades de aprendizaje exploratoria y explotadoras, obteniendo como resultados capacidades de gestión dinámica y un desempeño sostenible.

Uno de los factores que afectan a la cadena de suministros es la resiliencia, Según Fiksel (2006), como se citó en Pourhejazy, Kwon, Chang y Park (2017), la resiliencia es la capacidad de sobrevivencia y adaptación que tiene cualquier organización en entornos turbulentos. Asimismo, plantean que las principales determinantes de la resiliencia son la vulnerabilidad como catalizador de interrupciones y la capacidad para hacer resiliencia frente a las interrupciones.

\section{La competitividad}

La competitividad es un tema que tuvo una frecuencia de repetición en 6 publicaciones. La competitividad, según Sultan (2007), como se citó en Alsaad, Yousif y AlJedaiah (2018), es la capacidad que le permite a las empresas lograr sus objetivos propuestos. Asimismo, según Kavitha (2013), como se citó en Alsaad, Yousif y AlJedaiah (2018), depende de la habilidad para desempeñarse bien en las cuatro capacidades estratégicas que generan competitividad como son el costo, la calidad, la entrega y la flexibilidad".

Según Parola, Risitano, Ferretti y Panetti (2017), Barker, y otros (2021) los impulsores de la competitividad portuaria son: (1) economías de escala en el transporte marítimo, (2) cambios en la gobernanza portuaria, (3) cooperación entre puertos cercanos, (4) redes inter empresas, (5) desafíos ecológicos y de sostenibilidad.

\section{Innovación}

La innovación es un tema que tuvo una frecuencia de repetición en 6 publicaciones. Según Vaio (2018), el desarrollo sostenible requiere innovación en sostenibilidad y emprendedores enfocados. La innovación organizacional no es espontánea debe ser creada por la empresa en general y debe convertirse en parte del núcleo de sus actividades empresariales.

Según Gupta, Modgil, Gunasekaran y Bag (2020); Acciaro y Sys (2020); las presiones competitivas y regulatorias permiten enfatizar y controlar las cadenas de suministro ambientalmente sostenibles, asimismo, los requerimientos de las partes interesadas ejercen presión para que la empresa innove continuamente. "la innovación ha sido identificada como una de las principales vías para conciliar el crecimiento sectorial y las demandas ambientales” (p. 3).

Con respecto a la innovación en las cadenas de suministro, Gupta, Modgil, Gunasekaran, y Bag (2020); Pernas, Calatayud, y DeAngelis (2019) sostiene que, las industrias 4.0 están evolucionando como un marco prometedor hacia el avance de las actividades de fabricación y cadena de suministro tanto a nivel interno como externo, la industria 4.0, tiene como objetivo desarrollar capacidades dinámicas empresariales de sistemas ciber-físicos, computación cognitiva 
e internet de las cosas. Estas capacidades dinámicas se pueden utilizar para explotar sus actuales recursos o explorar nuevas formas de hacer negocios en la economía digital.

Según Pandey, Singh, Gunasekaran y Kaushik (2020), la industria 4.0 plantea mejoras en las capacidades digitales de todos los procesos de fabricación y de la cadena de suministros impulsando cambios revolucionarios en los dispositivos conectados, esforzándose para impulsar las fábricas inteligentes y permitir la fabricación avanzada. Asimismo, ha traído muchos desafíos para la seguridad de estos entornos digitales.

\section{Puertos}

Los puertos son un tema que tuvo una frecuencia de repetición en 6 publicaciones. Según Parola, Risitano, Ferretti y Panetti (2017), Wang y Mileski (2018), consideran a los puertos como redes empresariales dinámicas, cuyo valor en conjunto depende de las capacidades para crear recursos, competencias y capacidades en forma conjunta y cooperativa.

Según Chen, Cheung, Chu y Xu (2017), para seleccionar un centro de transbordo, hay tres aspectos a considerar. (1) las partes interesadas podrían preferir diferentes centros de transbordo en la logística global debido a su diferente función comercial. (2) se deben considerar al mismo tiempo las opciones de transporte marítimo como el aéreo, lo más relevante son los diversos aspectos del flujo de carga. (3) el país de origen de una empresa podría ser una preocupación en la estrategia de envío de las partes interesadas que influye en la elección de un puerto simplemente.

"En América Latina se observa que los principales puertos cuentan con terminales operadas por dos o más concesionarios, que compiten por la transferencia de carga del hinterland correspondiente. El sector naviero, por su parte, siempre ha presentado competencia entre múltiples operadores en las diversas rutas. Sin embargo, en años recientes se observa una creciente concentración gracias a varias fusiones entre navieras" (González, Serebrisky, 2021, p. 3).

\section{Grupos de Interés - Stakeholder}

Los grupos de interés es un tema que tuvo una frecuencia de repetición en 6 publicaciones. Según Valdez-Juárez, Gallardo-Vázquez y Ramos-Escobar (2018), la sostenibilidad se centra en acciones económicas sociales y ambientales, que le permiten sobrevivir y rentabilizarse a largo plazo, en el plano social se enfocan en los grupos de interés, compartiendo beneficios entre accionistas, empleados, clientes, proveedores.

\section{Transporte (de mercancías, marítimo, de carga)}

El transporte en conjunto tuvo una frecuencia de repetición en 15 publicaciones, siendo la frecuencia del transporte de mercancías de 6 publicaciones, el transporte marítimo 5 publicaciones y el transporte de carga 4 publicaciones. Según Agamez-Arias y Moyano-Fuentes (2017), la investigación sobre transporte intermodal tiene como principales líneas de investigación los siguientes: (1) Principios básicos del transporte intermodal, (2) Mejoras en la forma en que 
funcionan los sistemas de transporte intermodal, (3) Modelización de sistemas de transporte intermodal.

Los costos de transporte, que representan el componente principal de los costos no arancelarios según Anderson y Wincoop (2004), citado por Camisón-Haba y Clemente (2019) han caído en términos absolutos. Esta reducción en los costos de transporte por kilómetro se debe a las mejoras tecnológicas tanto en los medios de transporte como en la infraestructura de transporte, que han aumentado la eficiencia de la función de distribución física (Glaeser y Kohlhase (2004); citado por Camisón-Haba y Clemente (2019, p. 1), la participación del transporte en los costos de logística, sin embargo, había aumentado a casi el 44\% en 2002 (Davis y Drumm, 2002), citado por (Camisón-Haba y Clemente, 2019).

Los costos de transporte se han convertido en el principal obstáculo no arancelario al comercio; A menudo ocurre que la tasa efectiva de protección proporcionada por los costos de transporte incluso excede las protecciones creadas artificialmente, como lo ha demostrado empíricamente la Organización Mundial del Comercio (OMC) (2013) para EE. UU., por Micco y Pérez (2002, p. 43) citado por Camisón-Haba y Clemente (2019, p. 2).

\section{Gestión de la cadena de suministros verde}

La gestión de la cadena de suministros verde es un tema que tuvo una frecuencia de repetición en 4 publicaciones. Según Rahmani y Yavari (2019, como se citó en (Rahman, Ali, Moktadir y Kusi-Sarpong (2020), se define gestión de la cadena de suministro verde (GSCM) como "el proceso de utilizar insumos ecológicos o materiales ecológicos y convertir estos insumos en productos ecológicos que se pueden recuperar y reutilizar una vez finalizado su ciclo de vida, creando así un suministro prometedor y ecológico" (p. 673).

Según Srivastava (2007), sostiene que "la complejidad inherente de los problemas ambientales - sus múltiples partes interesadas, implicaciones inciertas para la competitividad y la importancia internacional - presentan desafíos importantes para los investigadores" (p. 70).

Según Srivastava (2007) la Gestión de la cadena de suministros Verde (GrSCM): puede reducir el impacto ecológico de la actividad industrial sin sacrificar la calidad, el costo, la confiabilidad, el rendimiento o la eficiencia en la utilización de energía. Implica un cambio de paradigma, pasando del control al final de la tubería para cumplir con las regulaciones ambientales a la situación no solo de minimizar el daño ecológico, sino también de generar ganancias económicas generales (p. 68).

La Integración de la Cadena de Suministro Verde (GSCI) para Lo, Zhang, Wang y Zhao (2018), es "el grado en que un fabricante colabora estratégicamente con sus socios de la cadena de suministro y gestiona de manera colaborativa los procesos intra e interorganizacionales para mejorar el desempeño ambiental" (p. 524).

Según Mudgal et al. (2010), como se citó en Balon (2019), "La barrera básica en la implementación de GSCM es la falta de voluntad para cambiar. La naturaleza del ser humana evita los cambios y GSCM necesita un cambio en la forma de pensar las prácticas”. La resistencia al 
cambio y la innovación de la fuerza laboral involucrada en diversas actividades, "actúa como una barrera significativa para GSCM" (p. 6).

Según Dubey, Gunasekaran, Papadopoulos, Childe, Shibin y Wamba (2017), los impulsores clave de la Gestión de la Cadena de Suministro Sostenible (SSCM) son: (1) Almacenamiento ecológico, (2) Colaboración estratégica con proveedores, (3) Conservación del medio ambiente, (4) Mejora continua, (5) Habilitación de tecnologías de la información, (6) Optimización Logística, (7) Presiones internas. (8) Presiones institucionales, (9) Valores sociales y ética, (10) Compromiso y estrategia corporativa, (11) Estabilidad económica y (12) Diseño de producto verde.

\section{Figura 8}

\section{Los objetivos de la sostenibilidad}

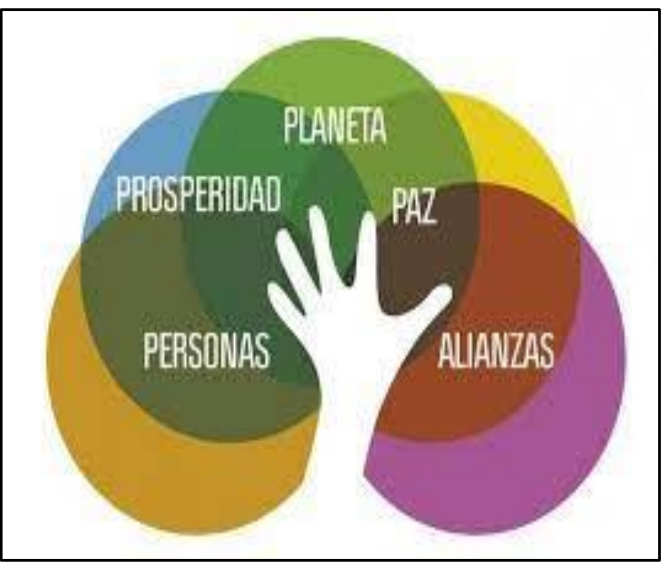

Fuente: (C) 2017 Catorce6. Todos los Derechos Reservados. Publicado: 13 octubre 2019. https://www.catorce6.com/actualidad-ambiental/internacional/17732-que-es-desarrollosostenible-y-sustentable. (Catorce6, 2019)

\section{Fuentes de mayor publicación e impacto}

En relación a las revistas que tienen más artículos publicados, vemos que las revistas que están orientadas al desarrollo sostenible son las que tienen mayor frecuencia de publicación; entre estas tenemos a Sustainability con 13 publicaciones, seguido por International Journal of Logistics Management con 7 publicaciones y Journal of Cleaner Production con 6 publicaciones.

En relación a las fuentes de mayor impacto, se observa que las revistas orientadas al desarrollo sostenible son las de mayor impacto. Entre estas, tenemos a Sustainability con hindex $=8$, seguido por International Journal of Logistics Management con h-index=5 y Journal of Cleaner Production con h-index $=5$.

Por lo tanto, podemos confirmar la tendencia observada anteriormente, ya que todas estas revistas están orientadas al desarrollo sostenible o a la gestión logística. 


\section{Conclusiones}

La globalización económica y el crecimiento del comercio mundial han producido un mayor interés por la competitividad de los operadores logísticos, debiendo considerar cada vez más los factores que influyen en los niveles de costos, calidad, velocidad, seguridad entre otros. En consecuencia, en el mundo académico se requiere una mayor investigación, con el fin de proponer soluciones a las demandas de competitividad de operadores logísticos.

Las grandes inversiones en las operaciones portuarias, así como, los riesgos que estas involucran, hacen que la gestión de los puertos, tengan cada vez mayor importancia en la investigación académica, cuya operatividad, competitividad, innovación e integración son las corrientes de investigación que se deben desarrollar a futuro. Estudiar a la competitividad de los operadores logísticos portuarios es un tema muy importante por los actores concurrentes, la cantidad de recursos que implican y el efecto que generan en las economías, por lo tanto, es necesario continuar y extender las futuras investigaciones en este campo de estudio.

El objetivo de la presente investigación fue realizar una revisión bibliométrica de la literatura científica sobre la competitividad de los operadores logísticos, que permita visualizar las tendencias de investigación, así como identificar los vacíos y discrepancias que se presentan. Al respecto, en las tendencias actuales sobre el desarrollo a nivel mundial, se observa que vienen adquiriendo mayor importancia el pilar social y ambiental, que conjuntamente con el económico están direccionando las investigaciones hacia la sostenibilidad en la gestión de la cadena de suministros, desarrollando de manera equilibrada los tres pilares de la sostenibilidad.

Teniendo en cuenta que los puertos y las zonas adyacentes son parte de los ecosistemas frágiles, los cuales puedes ser alterados por operaciones portuarias, que sólo están enfocadas en el resultado económico, se plantea que las futuras investigaciones se enfoquen en el desarrollo del pilar ambiental y social, lo cual permitirá una gestión sostenible y equilibrada que influirá positivamente en la competitividad de los operadores logísticos. Sin embargo, se debe tener en cuenta que las actividades desarrolladas por los operadores logísticos tienen como objetivo principal hacer negocios rentables, por lo tanto, no se debe descuidar el aspecto económico.

Considerando las necesidades y requerimientos de las partes involucradas (clientes proveedores, inversores, trabajadores, operadores, población afectada, entre otros), el desarrollo competitivo de los operadores logísticos tiene que enfocarse en la viabilidad de las inversiones, la equidad en el reparto de los beneficios y ser vivible para todos los involucrados, por lo tanto, las investigaciones sobre la innovación se deben desarrollar en esas direcciones.

Se han desarrollado investigaciones sobre cadena de suministros sostenible y cadena de suministro verde, sin embargo, no está clara la relación entre ambas. Se debe ampliar la investigación comparativa entre la gestión de la cadena de suministro sostenible y la gestión de la cadena de suministros verde con la finalidad de determinar la vinculación entre ambas si una incluye a la otra, si son complementarias o si se sustituyen. El aporte a la investigación se ha orientado en mejorar y ordenar la comprensión de los conceptos de la competitividad de los operadores logísticos. 
Este estudio se limitó a analizar la base de datos de Scopus del año 2017 al año 2021, por lo tanto, no se han considerado otras bases de datos que pueden ser relevantes. De esta revisión se obtuvieron 148 publicaciones que tratan sobre la competitividad de los operadores logísticos. considerando que no todas las publicaciones estuvieron disponibles se seleccionaron 50 publicaciones como base bibliográfica.

\section{Referencias bibliográficas}

Abushaikha, I., Al-Weshah, G., \& M., A. (2020). How do retail firms benefit from co-locating in logistics-intensive clusters? a focus on the inbound supply function. The International Review of Retail, Distribution and Consumer Research, Vol. 30.2, 27-45. https://doi.org/10.1080/09593969.2019.1635906

Acciaro, M., \& Sys, C. (2020). Innovation in the maritime sector: aligning strategy with outcomes. . Maritime Policy \& Management The flagship journal of international shipping and port research. https://www.tandfonline.com/action/showCitFormats?doi=10.108

Agamez-Arias, A. D. M., \& Moyano-Fuentes, J. (2017). Intermodal transport in freight distribution: a literature review. Transport Reviews, 37(6), 782-807. https://doi.org/10.1080/01441647.2017.1297868

Alsaad, A. K., Yousif, K. J., \& AlJedaiah, M. N. (2018). Collaboration: the key to gain value from IT in supply chain. EuroMed Journal of Business. https://doi.org/10.1108/EMJB-12-20170051

Ansari, Z. N., \& Kant, R. (2017). A state-of-art literature review reflecting 15 years of focus on sustainable supply chain management. Journal of cleaner production, 142, , 2524-2543. https://doi.org/10.1016/j.jclepro.2016.11.023

Balon, V. (2019). Green supply chain management: Pressures, practices, and performance An integrative literature review. Business Strategy And Development pag6. https://doi.org/10.1002/bsd2.91

Barker, A., Gibson, A., Hofer, A., Hofer, C., Moussaoui, I., \& Scott, M. (2021). A competitive dynamics perspective on the diversification of third-party logistics providers' service. Transportation Research Part E. https://doi.org/10.1016/j.tre.2020.102219

Belhadi, A., Venkatesh, M., Kamble, S., Rehman, S., \& Verma, S. (2021). A Artificial intelligence-driven innovation for enhancing supply chain resilience and performance under the effect of supply chain dynamism: an empirical investigation. Annals of Operations Research. https://doi.org/10.1007/s10479-021-03956-X

Belhadi, A.; Venkatesh, M.; Kamble, S.; Rehman, S.; Verma, S. (2021). A Artificial intelligence-driven innovation for enhancing supply chain resilience and performance under the effect of supply chain dynamism: an empirical investigation. Annals of Operations Research, 13. https://doi.org/10.1007/s10479-021-03956-x

Boojihawon, D., R. A., Liu, Y., \& Chicksand), D. (2020). Agile route-to-market distribution strategies in emerging markets: The case of Paraguay. Journal of International Management. https://doi.org/10.1016/j.intman.2020.100740

Botero, L. (julio-diciembre, de 2014). Internacionalización y Competitividad. Ciencias Estratégicas, vol. 22, núm. 32; Universidad Pontificia Bolivariana, 187-196. Obtenido de https://www.redalyc.org/articulo.oa?id=151339264001 
Calatayud, A., y Montes, 1. (2021). Logísticaen América Latina y el Caribe:oportunidades, desafíos y líneas de acción. Biblioteca Felipe Herrera del Banco Interamericano de Desarrollo. https://doi.org/10.18235/0003278

Camisón-Haba, S., \& Clemente, J. (2019). A global model for the estimation of transport costs. Journal of Economic Research-Ekonomska Istraživanja. https://doi.org/10.1080/1331677X.2019.1584044

Catorce6, R. A. (13 de Octubre de 2019). Catorce6. Obtenido de Catorce6: https://www.catorce6.com/actualidad-ambiental/internacional/17732-que-es-desarrollosostenible-y-sustentable

Chen, G., Cheung, W., Chu, S. C., \& Xu, L. (2017). Transshipment Hub Selection From a Shipper's and Freight Forwarder's Perspective. Expert systems with applications, 83, 396-404. https://doi.org/10.1016/j.eswa.2017.04.044

Consejo Nacional de Competitividad. (15 de Junio de 2018). Índice de Desempeño Logístico 2018. 3. Recuperado el 2021, de http://www.competitividad.org.do/wpcontent/uploads/2018/07/\%C3\%8Dndice-de-Desempe\%C3\%B1o-Log\%C3\%ADstico2018-Final.pdf

De-Oliveira, U. R., Espindola, L. S., da Silva, I. R., da Silva, I. N., \& Rocha, H. M. (2018). SA systematic literature review on green supply chain management: .Research implications and future perspectives. Journal of Cleaner Production, 187,, 537-561. https://doi.org/10.1016/j.jclepro.2018.03.083

Di Vaio A., \&. V. (2018). Management Innovation for Environmental Sustainability in Seaports: Managerial Accounting Instruments and Training for Competitive Green Ports beyond the Regulations. Sustainability MDPI, 35. https://doi.org/10.3390/su10030783

Di Vaio, A., Varriale, L., \& \& Alvino, F. (2018). Key performance indicators for developing environmentally sustainable and energy efficient ports: Evidence from Italy. Energy policy, 122,, 229-240. https://doi.org/10.1016/j.enpol.2018.07.046

Dubey, R., Gunasekaran, A., Childe, S. J., Papadopoulos, T., \& Wamba, S. F. (2017). World class sustainable supply chain management: critical review and further research directions. The International Journal of Logistics Management.

Dubey, R., Gunasekaran, A., Childe, S., Papadopoulos, T., \& \& Fosso-Wamba, S. (2017). WorLd class sustainable supply chain management: critical review and further research directions. The International Journal of Logistics Management, , 28(2), 232-362. https://doi.org/10.1108/IJLM-07-2015-0112

Dubey, R., Gunasekaran, A., Papadopoulos, T., Childe, S. J., Shibin, K. T., \& Wamba, S. F. (2017). Sustainable supply chain management: framework and further research directions. Journal of cleaner production, 142, , 1119-1130.

Evangelista, P., Santoro, L., \& Thomas, A. (2018). Environmental sustainability in third-party logistics service providers: A systematic literature review from 2000-2016. Sustainability, 10(5), 1627. https://doi.org/10.3390/su10051627

Gani, A. (2017). The Logistics Performance Effect in International Trade. The Asian Journal of Shipping and Logistics, 33(4), , 279-288. https://doi.org/10.1016/j.ajs1.2017.12.012

Giordano, P., Campos, R., Ortiz, C., Michalczewsky, K., y DeAngelis, J. (2018). La sofisticación de las exportaciones como motor del crecimiento. Monitor de comercio e integración $N^{\circ}$ IDB-MG-673, 80. http://creativecommons.org/licenses/by-nc-nd/3.0/igo/legalcode 
González, A.; Serebrisky. (2021). Libre competencia en agua, energía y transporte - La experiencia de América Latina entre 2010 y 2020. Banco Interamericano de Desarrollo, Nota técnica $N^{\circ}$ IDB-TN-02104 pág 3.

Granillo-Macías, R. G.-H.-R. (2019). Operadores logísticos. Ingenio Y Conciencia Boletín Científico De La Escuela Superior Ciudad Sahagún, 6(11), 44-48,. https://doi.org/10.29057/ess.v6i11.3740

Gupta, S., Modgil, S., Gunasekaran, A., \& Bag, S. (2020). Dynamic capabilities and institutional theories for Industry 4.0 and digital supply chain. In. Supply Chain Forum: An International Journal 21(3), 139-157. https://doi.org/10.1080/16258312.2020.1757369

Jian, J., Guo, Y., Jiang, L., An, Y., \& Su, J. (2019). A multi-objective optimization model for green supply chain considering environmental benefits. Sustainability, 11(21), 5911. https://doi.org/10.3390/su11215911

Klumpp, M. (2018). Automation and artificial intelligence in business logistics systems: human reactions and collaboration requirements. International Journal of Logistics Research and Applications, 21(3), 224-242. https://doi.org/10.1080/13675567.2017.1384451

Knop, K. (2019). Evaluation of quality of services provided by transport \& logistics operator from pharmaceutical industry for improvement purposes. Transportation Research Procedia, 40, 1080-1087. https://doi.org/10.1016/j.trpro.2019.07.151

Lo, S. M., Zhang, S., Wang, Z., \& Zhao, X. (2018). The impact of relationship quality and supplier development on green supply chain integration: A mediation and moderation analysis. Journal of cleaner production, 202, 524-535. https://doi.org/10.1016/j.jclepro.2018.08.175

Mohammadi, E., Talaie, H., \& Safari, H. (2018). Supplier evaluation and selection for sustainable supply chain management under uncertainty conditions. International Journal of Sustainable Engineering. International Journal of Sustainable Engineering. https://doi.org/10.1080/19397038.2017.1421277

Notteboom, T., Parola, F., Satta, G., \& Risitano, M. (2017). A taxonomy of logistics centres: overcoming conceptual ambiguity. Transport reviews, 37(3), 276-299. https://doi.org/10.1080/01441647.2016.1231234

Pandey, S., Singh, R. K., Gunasekaran, A., \& Kaushik, A. (2020). Cyber security risks in globalized supply chains: conceptual framework. Journal of Global Operations and Strategic Sourcing. https://doi.org/10.1108/JGOSS-05-2019-0042

Parola, F., Pallis, A. A., Risitano, M., \& Ferretti, M. (2018). Marketing strategies of Port Authorities: A multi-dimensional theorisation. Transportation Research Part A: Policy and Practice, 111, , 199-212. https://doi.org/10.1016/j.tra.2018.03.012

Parola, F., Risitano, M., Ferretti, M., \& Panetti, E. (2017). The drivers of port competitiveness: a critical review. Transport Reviews, 37(1), 116-138. https://doi.org/10.1080/01441647.2016.1231232

Pernas, M., Calatayud, A., y DeAngelis, J. (2019). La marcha se acelera: los vehículos del comercio. Banco Interamericano de Desarrollo, Nota técnica $N^{\circ}$ IDB-TN-1768, 5. http://creativecommons.org/licenses/by-nc-nd/3.0/igo/legalcode

Pourhejazy, P., Kwon, O. K., Chang, Y. T., \& Park, H. K. (2017). Evaluating resiliency of supply chain network: A data envelopment analysis approach. Sustainability, 9(2), 255. https://doi.org/10.3390/su9020255

Rahman, T., Ali, S. M., Moktadir, M. A., \& Kusi-Sarpong, S. (2020). Evaluating barriers to implementing green supply chain management: An example from an emerging economy. 
$\begin{array}{lllll}\text { Production } \quad \text { Planning } \quad \& \quad \text { Control, } & \text { 31(8), }\end{array}$ https://doi.org/10.1080/09537287.2019.1674939

Regional, P. Z. (6 de Agosto de 2019). Publicado por regionalpymezonasur. Obtenido de Publicado por regionalpymezonasur. https://www.regionalpymezonasur.org.ar/desarrollosostenible/

Russell, E., Lee, J., \& Clift, R. (2018). Can the SDGs provide a basis for supply chain decisions in the construction sector?. Sustainability, 10(3), 629. https://doi.org/10.3390/su10030629

Sibel, Y., \& Bulent, S. (2019). Effects of green supply chain management practices on sustainability performance. Journal of Manufacturing Technology Management., 98. https://doi.org/10.1108/JMTM-03-2018-0099

Srivastava, S. K. (2007). Green supply-chain management: a state-of-the-art literature review. International journal of management reviews, 9(1), 53-80.

Vaio, A. D. (2018). Management innovation for environmental sustainability in seaports: Managerial accounting instruments and training for competitive green ports beyond the regulations. Sustainability, 10(3), 1-35.

Valdez-Juárez, L. E., Gallardo-Vázquez, D., \& Ramos-Escobar, E. A. (2018). CSR and the Supply Chain: Effects on the Results of SMEs. Sustainability, 10(7),, 2356. https://doi.org/10.3390/su10072356

Wang, P., \& Mileski, J. (2018). Strategic Maritime Management as a New Emerging Field in Maritime Studies. Journal of Sustainable Engineering. https://doi.org/10.1108/MABR-062018-0019

Xiao, Z., Yuan, Q., Sun, Y., \& \& Sun, X. (2021). New paradigm of logistics space reorganization: E-commerce, land use, and supply chain management. Transportation Research Interdisciplinary Perspectives, 9, 100300 - 100310. https://doi.org/10.1016/j.trip.2021

Yang, Y., Jia, F., \& Xu, Z. (2019). Towards an integrated conceptual model of supply chain learning: an extended resource-based view. Supply Chain Management. An International Journal, 24(2), 189-214. https://doi.org/10.1108/SCM-11-2017-0359

Zheng, Y., Zhao, J., \& Shao, G. (2020). Port City Sustainability: A Review of Its Research Trends. Sustainability. https://doi.org/10.3390/su12208355 